\title{
Optimization of EOQ model with space constraint: An intuitionistic fuzzy geometric programming approach
}

\section{Bappa Mondal ${ }^{1}$, Arindam Garai ${ }^{2, *}$ and Tapan Kumar Roy ${ }^{3}$}

${ }^{1}$ Department of Mathematics, Indian Institute of Engineering Science and Technology Shibpur, Howrah-711103, West Bengal, India e-mail: bappa802@gmail.com

${ }^{2}$ Department of Mathematics, Sonarpur Mahavidyalaya Sonarpur, Kolkata-700149, West Bengal, India e-mail: fuzzy_arindam@yahoo.com

* Corresponding author

${ }^{3}$ Department of Mathematics, Indian Institute of Engineering Science and Technology Shibpur, Howrah-711103, West Bengal, India e-mail: roy_t_k@yahoo.co.in

Received: 20 May $2018 \quad$ Revised: 26 September $2018 \quad$ Accepted: 28 September 2018

\begin{abstract}
In this paper, we present a deterministic single objective economic order quantity (EOQ) model with space constraint in intuitionistic fuzzy environment. Here we take variable limit production cost, time dependent holding cost into account. We propose intuitionistic fuzzy geometric programming by extending existing fuzzy geometric programming to solve non-linear optimization problems. Next, we minimize the total average cost (TAC) of proposed EOQ model by applying intuitionistic fuzzy geometric programming. We consider one numerical application to show that the optimal solution of the proposed model by intuitionistic fuzzy geometric programming is more preferable than that of crisp and fuzzy geometric programming. Also we perform sensitivity analysis of parameters and present key managerial insights. Finally, we draw the conclusions.
\end{abstract}

Keywords: Economic order quantity, Geometric programming, Intuitionistic fuzzy geometric programming, Max-additive operator, Shape parameter, Storage space.

2010 Mathematics Subject Classification: 03E72, 90C30, 90C70. 


\section{Introduction}

Inventory can be defined as an idle resource of any enterprise. Although idle, a certain amount of inventory is essential for smooth conduction of organizational activities. Control of inventory is one of the key areas for operational management. An adequate control of inventory can significantly bring down operating costs and increase efficiency. In order to minimize the total costs of inventory e.g., holding costs, order costs, and shortage costs, one needs to determine Economic Order Quantity (EOQ). The concept of EOQ was first developed by F. Harris [12] and subsequently applied by Wilson. Hoon Jung et al. [17, 18] discussed optimal inventory policies for maximizing profit of EOQ models under various cost functions. N. K. Mondal [23] considered a model with deteriorating items. S. Islam [13] formulated multi-objective inventory model with capacity constraint and shortage cost. S. Sadjadi et al. [29] considered a model with cubic demand function. L. Janseen [16] presented one extensive literature review on deteriorating inventory models. In recent era, we find that the development of EOQ model is based on several constraints, among which budgetary and space constraints have generated considerable attention of researchers. In this paper, we consider a non-linear EOQ model with space constraint.

We know that one of the most popular and constructive method to solve Non-Linear Programming (NLP) problem is Geometric Programming (GP). The method is useful in applications of a variety of optimization problems and falls under the general class of signomial problems. It can be used to solve large scale, real life based problems by quantifying them into an equivalent optimization model. Another major advantage of using GP is that it allows sensitivity analysis to be performed efficiently [3]. Historically, through the investigation of cost minimization techniques for engineering and designing problem $[34,35]$ Zenner introduced the notion of GP. Later, Duffin et al. $[9,10]$ presented the mathematical formulations of GP. Kochenberger [20] was the first scientist to explain non-linear EOQ problem by GP. Beightler et al. [3] studied advantages of applying GP in real life based problems. Afterwards, Cheng [6, 7] formulated an EOQ model with unit production cost. Lee [21] proposed GP formulations for optimal order quantities and prices with storage space limitations. Sadjadi et al. [24, 28] investigated the integrated pricing, lot sizing and marketing planning model and reviewed literature of last two decades. Tabatabaei et al [31] discussed optimal pricing and marketing planning for deteriorating items.

On the other hand, in real life based imprecise environment, total inventory cost of an EOQ model is controlled by several constraints. We can find many restrictions that can affect the optimal inventory cost, e.g. ceiling on storage space, number of orders and production cost etc. In this paper, we consider only the ceiling on storage space to be imprecise in nature. Impreciseness in mathematics was first considered by Zadeh [33] with the introduction of fuzzy set theory. Afterwards, Bellman and Zadeh [4] used fuzzy set theory in decision making problems. Tanaka et al. [32] proposed objectives as fuzzy goals and Zimmerman [36] presented solution method for multi-objective linear programming problems in fuzzy environment. In subsequent years, mathematicians have applied fuzzy set theory in different directions. Sommer [30] employed fuzzy concept to inventory and production-scheduling problem. Park [25] examined EOQ model in fuzzy environment. Roy and Maiti [27] solved single objective EOQ model using GP technique in fuzzy environment. Islam and Mandal [14] formulated one fuzzy economic production quantity 
(EPQ) model with flexibility and reliability considerations. G.S. Mahapatra et al. [22] considered an EPQ model with fuzzy and solved it by parametric GP technique.

In fuzzy set theory, we consider the degree of membership function for objective functions and constraints. In recent years, fuzzy set theory has been widely developed and several modifications have appeared. Atanassov [2] developed Intuitionistic Fuzzy (IF) set theory, where we consider non-membership function along with membership function for imprecise information. Angelov [1] developed optimization technique in IF environment. Pramanik and Roy [26] analyzed vector operational problem using IF goal programming. A transportation model was elucidated by Jana and Roy [15] by using multi-objective IF linear programming. Chakrabortty et al. [5] applied IF optimization technique for Pareto optimal solution of manufacturing inventory models with shortages. Dey and Roy [8] employed IF optimization technique in two bar truss design problem. Kaur and Rachana [19] presented methodology and numerical solution for vendor selection problem using IF technique. Garai et al. [11] studied optimization method in IF environment and introduced one new set. Although we have performed extensive literature review and have found different types of EOQ models in IF environment, we observe that these models have been solved by using various software packages. In this paper, we consider one EOQ model with space constraint. We solve the proposed model by using GP technique in IF environment.

The rest of the paper is organized as follows. In Section 2, we present some elementary definitions. In Section 3, we construct one single objective EOQ model with space constraint. In Section 4, we solve the proposed model in crisp environment by applying GP. Next in Section 5, we present optimal solution of proposed model by applying fuzzy GP. In Section 6, we present IF GP and solve the proposed model in IF environment. In Section 7, we present sensitivity analysis and discussed key managerial insights. Finally in Section 8, we draw the conclusion and present future scopes of research.

\section{Preliminaries}

Definition 2.1 [2]. Let $X$ be an universal set. An IF set $A$ in $X$ is an object of the form $A=$ $\left\{\left\langle x, \mu_{A}(x), \nu_{A}(x)\right\rangle \mid x \in X\right\}$. Here and thereafter, $\mu_{A}(x): X \rightarrow[0,1]$ and $\nu_{A}(x): X \rightarrow[0,1]$ are the membership function and non-membership function of $A$, respectively, and satisfy the condition $0 \leq \mu_{A}(x)+\nu_{A}(x) \leq 1, \forall x \in X$.

Definition 2.2 [2]. Let $X$ be an universal set and $A$ and $B$ be any two subsets of $X$. Then the union of $A$ and $B$ is denoted by $A \cup B$ and is defined as

$$
A \cup B=\left\{\left\langle x, \max \left(\mu_{A}(x), \mu_{B}(x)\right), \min \left(\nu_{A}(x), \nu_{B}(x)\right)\right\rangle \mid x \in X\right\} .
$$

Definition 2.3 [2]. Let $X$ be an universal set and $A$ and $B$ be any two subsets of $X$. Then the intersection of $A$ and $B$ is denoted by $A \cap B$ and is defined as

$$
A \cap B=\left\{\left\langle x, \min \left(\mu_{A}(x), \mu_{B}(x)\right), \max \left(\nu_{A}(x), \nu_{B}(x)\right)\right\rangle \mid x \in X\right\} .
$$

Definition 2.4 [2]. Let $X$ be an universal set and $A$ be any subset of $X$. Then the complement of $A$ is denoted by $A^{c}$ and is defined as $A^{c}=\left\{\left\langle x, \nu_{A}(x)\right\rangle \mid x \in X\right\}$. 


\section{Single objective EOQ model with space constraint}

In this paper, we consider one single objective EOQ model with space constraint. Here the unit production cost is as follows

$$
P(D, S)=\theta D^{-x} S^{-1}
$$

We can note that the shape parameter $(x)$ must lie within predetermined values so that to satisfy the positivity conditions of the Dual Geometric Programming Problem (DGPP). The following notations are used: $D$ - Demand per unit time, which is constant; $H(t)$ - Holding cost per unit item, which is time $(t)$ dependent; $I(t)$ - Inventory level at any time, $t \geq 0 ; P(D, S)$ - Unit demand $(D)$ and set-up cost $(S)$ dependent production cost; $Q$ - Production quantity per batch; $S$ - Set-up cost per unit time; $T$ - Period of cycle; $T A C(D, S, Q)$ - Total average cost per unit time; $W$ - Total storage space area; $w_{0}-$ Space area per unit quantity.

We develop a single objective inventory model with these notations and the following assumptions.

\subsection{Assumptions}

To specify scopes of study and to further simplify the proposed EOQ model, we hypothesize the following assumptions:

(i) the proposed EOQ model involves only one item;

(ii) replenishment occurs instantaneously at infinite rate;

(iii) lead time is negligible;

(iv) demand rate is constant;

(v) holding cost is time dependent $(H(t)=$ "at");

(vi) upgrade to modern machineries involves higher costs, which is considered to be a part of the set-up cost. But these machineries have higher production rates and other advantages. So, large scale production can bring down the unit production cost and it is generally adopted when demand is high. Hence, the unit production costs become inversely proportional to the set-up costs and inversely related to the rate of demand, i.e.,

$$
P(D, S)=\theta D^{-x} S^{-1}, \quad \theta, x \in R^{+}
$$

(vii) shortage is not allowed.

\subsection{Formulation of the model}

In this paper, we assume that the initial inventory level (i.e., $t=0$ ) is $Q$. Also we assume that the inventory level gradually decreases in $[0, T]$ and it becomes zero at time $T$. Since shortage is not allowed, we find that the cycle is repeated over time period $T$. We illustrate the proposed inventory model graphically in Fig. 1. 


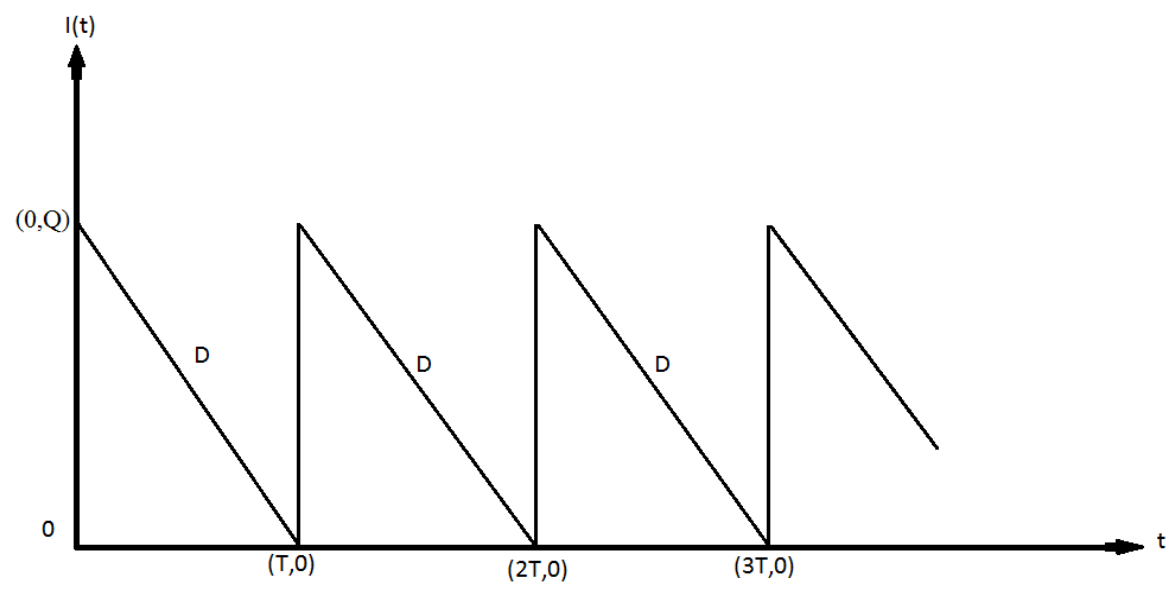

Figure 1: Inventory model

Let the inventory level at any time $t$ in $[0, T]$ be denoted by $Q(t)$. Consequently, the differential equation for instantaneous inventory level $Q(t)$ at time $t$ in $[0, T]$ is as follows:

$$
\frac{d I(t)}{d t}=-D \quad \text { for } 0 \leq t \leq T
$$

with boundary conditions as $I(0)=Q, I(T)=0$.

Using the given conditions, we find as follows

$$
I(t)=D(T-t)
$$

Therefore, the inventory holding cost is as follows

$$
\int_{0}^{T} H(t) I(t) d(t)=\frac{a Q^{3}}{6 D^{2}} .
$$

Hence, we find total average inventory cost per cycle $[0, T]$ as follows

$$
T A C(D, S, Q)=\frac{S D}{Q}+\frac{a Q^{2}}{6 D}+\theta D^{1-x} S^{-1}
$$

Here we consider that the maximum floor space for storing items in a warehouse is $W$. Therefore, storage area $w_{0} Q$ for production quantity $Q$ cannot exceed maximum floor space in warehouse for storing items in any time $t$. Therefore, the space constraint is as follows

$$
w_{0} Q \leq W
$$

Hence, the inventory model in crisp environment is as follows

$$
\min T A C(D, S, Q)=\frac{S D}{Q}+\frac{a Q^{2}}{6 D}+\theta D^{1-x} S^{-1}
$$

such that

$$
\begin{aligned}
& S(Q) \equiv w_{0} Q \leq W \\
& D, S, Q>0 .
\end{aligned}
$$




\section{Solution of the inventory model by classical GP}

We solve the proposed model by applying GP. Here we find that the degree of difficulty (DD) is 0. By applying Duffin and Peterson theorem [10] of GP on equation (1), we obtain the DGPP as follows

$$
\begin{aligned}
& \operatorname{Max} d(\mathrm{w})=\left(\frac{1}{w_{01}}\right)^{w_{01}}\left(\frac{a}{6 w_{02}}\right)^{w_{02}}\left(\frac{\theta}{w_{03}}\right)^{w_{03}}\left(\frac{w_{0}}{W w_{11}}\right)^{w_{11}} w_{11} w_{11} \\
& \text { such that } \\
& w_{01}+w_{02}+w_{03}=1 \\
& w_{01}-w_{02}+(1-x) w_{03}=0, \\
& w_{01}-w_{03}=0 \\
& -w_{01}+2 w_{02}+w_{11}=0 \\
& w_{01}, w_{02}, w_{03}, w_{11} \geq 0 .
\end{aligned}
$$

Hence, in crisp environment, we find the optimal solution as follows

$$
w_{01}^{*}=w_{03}^{*}=\frac{1}{4-x}, w_{02}^{*}=\frac{2-x}{4-x}, \quad w_{11}^{*}=\frac{2 x-3}{4-x} .
$$

Here we note that $x$ must lie in the interval $[1.5,2]$ so that all the dual variables can remain positive. Thus the optimal values of the primal variables are as follows

$$
\begin{aligned}
D^{*} & =\left\{\frac{1}{\theta}\left(\frac{a}{6(2-x)}\right)^{2}\left(\frac{W}{w_{0}}\right)^{5}\right\}^{\frac{1}{4-x}}, \\
S^{*} & =\left\{\left(\frac{\theta W}{w_{0}}\right)^{2}\left(\frac{6 w_{0}^{3}(2-x)}{a W^{3}}\right)^{x}\right\}^{\frac{1}{4-x}}, \\
Q^{*} & =\frac{W}{w_{0}}
\end{aligned}
$$

with optimal TAC as follows

$$
T A C^{*}\left(D^{*}, S^{*}, Q^{*}\right)=(4-x)\left\{\theta\left(\frac{w_{0}}{W}\right)^{(2 x-3)}\left(\frac{a}{6(2-x)}\right)^{(2-x)}\right\}^{\frac{1}{4-x}}=T_{1} \text { (say). }
$$

\section{Solution of the inventory model by fuzzy GP}

We employ the max-additive operator to solve the proposed EOQ model in a fuzzy environment. Here we compute the individual minimum and maximum values for an objective function TAC and space constraint of model (1), as given in Table 1. The goal and goal plus tolerance values for the membership functions of objective function and constraint are supplied by the decision maker (DM). 


\begin{tabular}{|c|c|c|}
\hline $\begin{array}{c}\text { Demand per } \\
\text { unit time }(D)\end{array}$ & $\left\{\frac{1}{\theta}\left(\frac{a}{6(2-x)}\right)^{2}\left(\frac{W}{w_{0}}\right)^{5}\right\}^{\frac{1}{4-x}}$ & $\left\{\frac{1}{\theta}\left(\frac{a}{6(2-x)}\right)^{2}\left(\frac{W+w_{p}}{w_{0}}\right)^{5},\right\}^{\frac{1}{4-x}}$ \\
\hline $\begin{array}{c}\text { Set up cost } \\
(S)\end{array}$ & $\left\{\left(\frac{\theta W}{w_{0}}\right)^{2}\left(\frac{6 w_{0}^{3}(2-x)}{a W^{3}}\right)^{x}\right\}^{\frac{1}{4-x}}$ & $\left\{\left(\frac{\theta\left(W+w_{p}\right)}{w_{0}}\right)^{2}\left(\frac{6 w_{0}^{3}(2-x)}{a\left(W+w_{p}\right)^{3}}\right)^{x}\right\}^{\frac{1}{4-x}}$ \\
\hline $\begin{array}{c}\text { Production quantity } \\
\text { per batch }(Q)\end{array}$ & $\frac{W}{w_{0}}$ & $\frac{W+w_{p}}{w_{0}}$ \\
\hline $\begin{array}{c}\text { Total Average Cost } \\
T A C(D, S, Q)\end{array}$ & $(4-x)\left\{\theta\left(\frac{w_{0}}{W}\right)^{(2 x-3)}\left(\frac{a}{6(2-x)}\right)^{(2-x)}\right\}^{\frac{1}{4-x}}$ & $(4-x)\left\{\theta\left(\frac{w_{0}}{W+w_{p}}\right)^{(2 x-3)}\left(\frac{a}{6(2-x)}\right)^{(2-x)}\right\}^{\frac{1}{4-x}}$ \\
\hline
\end{tabular}

Table 1: Individual maximum and minimum values of decision variables and TAC

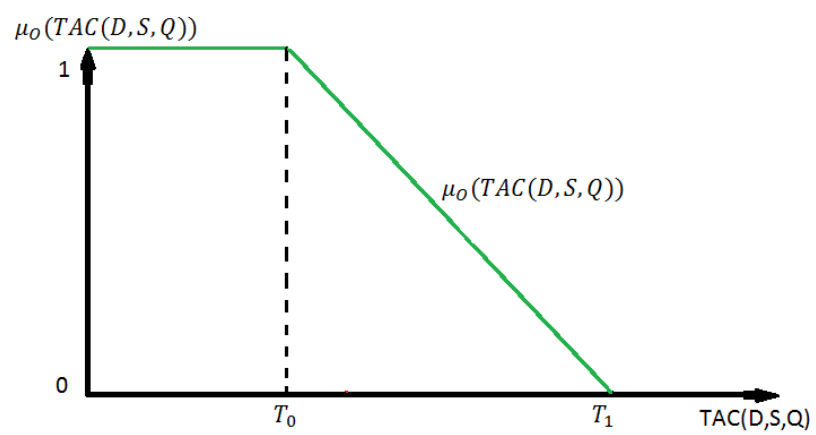

Figure 2: Membership function of a fuzzy objective function

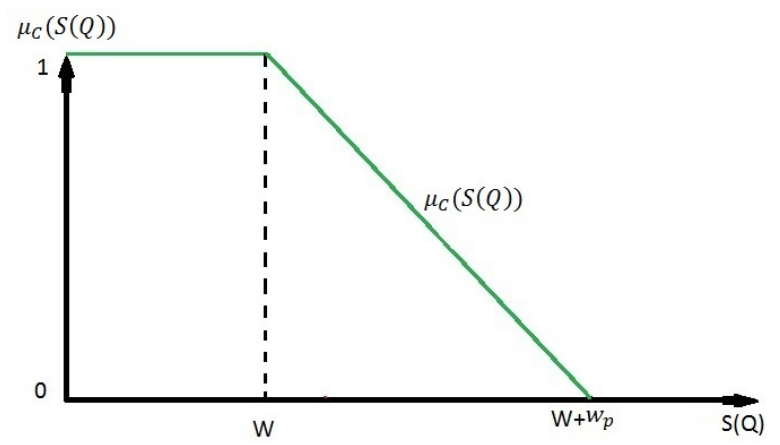

Figure 3: Membership function of a fuzzy constraint

Here, for the sake of simplicity, we consider a linear membership function for TAC and space constraint (graphically it has been presented in Figure 2 and Figure 3) as follows:

$$
\begin{gathered}
\mu_{\tilde{O}}(T A C(D, S, Q))= \begin{cases}1 & \text { if } T A C(D, S, Q) \leq T_{0} \\
\frac{T_{1}-T A C(D, S, Q)}{T_{1}-T_{0}} & \text { if } T_{0} \leq T A C(D, S, Q) \leq T_{1} \\
0 & \text { otherwise. }\end{cases} \\
\mu_{\tilde{C}}(S(Q))= \begin{cases}1 & \text { if } w_{0} Q \leq W \\
\frac{W+w_{p}-w_{0} Q}{w_{p}} & \text { if } W \leq w_{0} Q \leq W+w_{p} \\
0 & \text { otherwise. }\end{cases}
\end{gathered}
$$


Next we formulate the proposed model as follows

$$
\begin{aligned}
& \max \left\{\mu_{\tilde{O}}(T A C(D, S, Q)) \mu_{\tilde{C}}(S(Q))\right\} \\
& \text { such that } \\
& 0<\mu_{\tilde{O}}(T A C(D, S, Q))+\mu_{\tilde{C}}(S(Q))<1, \\
& D, S, Q>0
\end{aligned}
$$

By applying the max-additive operator, we convert model (1) to a crisp Primal Geometric Programming Problem (PGPP). To reduce DD, we can use convex combination operator and obtain as follows:

$$
\max V F_{F A}(D, S, Q)=F_{K}-V F_{F A 1}(D, S, Q) .
$$

Here $F_{K}=\frac{T_{0}}{T_{1}-T_{0}}+\frac{W+w_{p}}{w_{p}}$ and $V F_{F A 1}(D, S, Q)=\frac{T A C(D, S, Q)}{T_{1}-T_{0}}+\frac{w_{0} Q}{w_{p}}$. Therefore, the model reduces as follows:

$$
\begin{aligned}
& \min V F_{F A 1}(D, S, Q)=\frac{S D}{Q\left(T_{1}-T_{0}\right)}+\frac{a Q^{2}}{6 D\left(T_{1}-T_{0}\right)}+\frac{\theta D^{1-x}}{\left(T_{1}-T_{0}\right) S}+\frac{w_{0} Q}{w_{p}} \\
& \text { such that } \\
& D, S>0, Q \in\left[\frac{W}{w_{0}}, \frac{W+w_{p}}{w_{0}}\right], T A C(D, S, Q) \in\left[T_{0}, T_{1}\right] .
\end{aligned}
$$

It is an unconstrained PGPP with $\mathrm{DD}=0$. Hence, the optimal values for the primal variables of model (2) are as follows:

$$
\begin{aligned}
& D^{*}=\frac{3}{2}\left\{\theta 6^{(x-2)}\left(\frac{a}{2-x}\right)^{3}\left(\frac{w_{0}}{w_{p}}\right)^{(2 x-3)}\left(\frac{2 x-3}{T_{1}-T_{0}}\right)^{5}\right\}^{\frac{1}{x+1}}, \\
& S^{*}=2\left\{\theta 6^{(x-2)}\left(\frac{T_{1}-T_{0}}{2 x-3}\right)^{(3 x-2)}\left(\frac{w_{0}}{w_{p}}\right)^{(2 x-3)}\left(\frac{a}{2-x}\right)^{(1-2 x)}\right\}^{\frac{1}{x+1}}, \\
& Q^{*}=3(2 x-3)\left\{\theta\left(\frac{1}{T_{1}-T_{0}}\right)^{(4-x)}\left(\frac{a}{6(2-x)}\right)^{(2-x)}\left(\frac{w_{0}}{w_{p}(2 x-3)}\right)^{(2 x-3)}\right\}^{\frac{1}{x+1}},
\end{aligned}
$$

with optimal TAC as follows

$$
\begin{aligned}
\operatorname{TAC}^{*}\left(D^{*}, S^{*}, Q^{*}\right)= & {\left[\left\{\left(\frac{a}{6(2-x)}\right)^{(2-x)}\left(\frac{w_{0}\left(T_{1}-T_{0}\right)}{w_{p}(2 x-3)}\right)^{(2 x-3)}\right\}^{\frac{1}{x+1}}\right.} \\
& \left.\left\{1+\left(\frac{2-x}{6^{x}}\right)^{\frac{1}{x+1}}+\left(\frac{w_{p}}{3 w_{0}}\right)^{(2 x-3)}\right\}\right]
\end{aligned}
$$

provided $Q^{*} \in\left[\frac{W}{w_{0}}, \frac{W+w_{p}}{w_{0}}\right], T A C^{*}\left(D^{*}, S^{*}, Q^{*}\right) \in\left[T_{0}, T_{1}\right]$.

\section{Solution of the inventory model by IFGP}

Next we employ the IF optimization method to solve the proposed EOQ model (1). We can obtain goal and goal plus tolerance values for non-membership functions of TAC and space constraint 


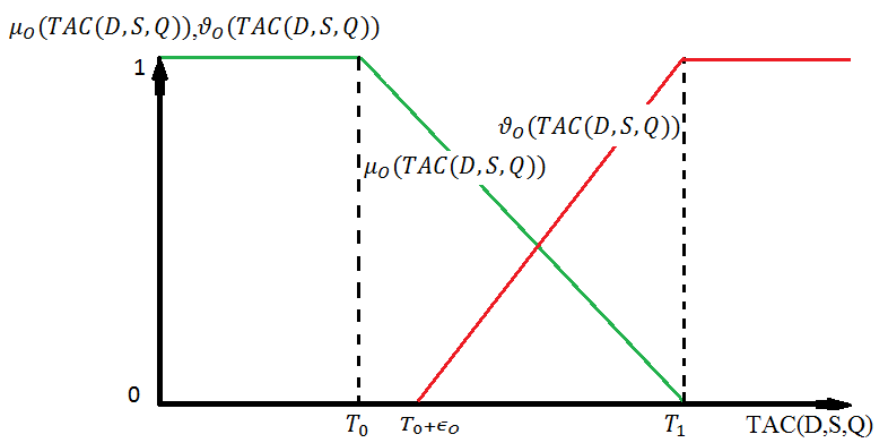

Figure 4: Membership and non-membership function of the IF objective function

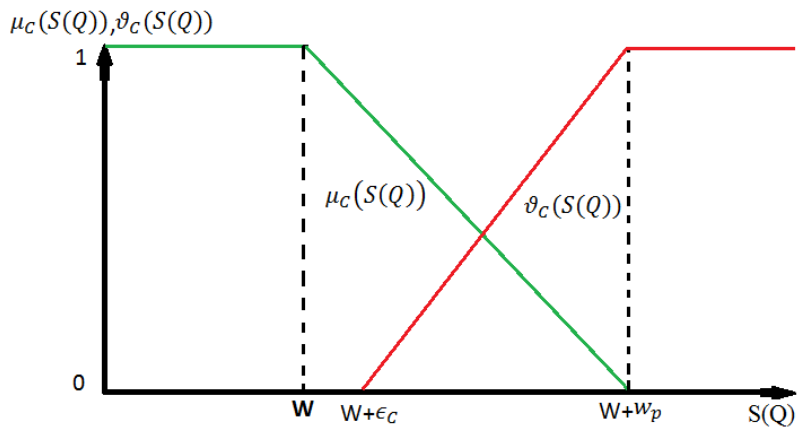

Figure 5: Membership and non-membership function for the IF constraint

from DM. We note that these values must lie within the respective maximum and minimum values of TAC and space constraint in Table 1.

Based on those values, we construct the following linear non-membership functions of TAC and space constraint (See Figure 4 and Figure 5) as follows:

$$
\begin{gathered}
\nu_{\tilde{O}}(T A C(D, S, Q))= \begin{cases}0 & \text { if } T A C(D, S, Q) \leq T_{0}+\epsilon_{0} \\
\frac{T A C(D, S, Q)-T_{0}-\epsilon_{0}}{T_{1}-T_{0}-\epsilon_{0}} & \text { if } T_{0}+\epsilon_{0} \leq T A C(D, S, Q) \leq T_{1} \\
1 & \text { otherwise. }\end{cases} \\
\nu_{\tilde{C}}(S(Q))= \begin{cases}0 & \text { if } w_{0} Q \leq W+\epsilon_{C} \\
\frac{w_{0} Q-W-\epsilon_{C}}{w_{p}-\epsilon_{C}} & \text { if } W+\epsilon_{C} \leq w_{0} Q \leq W+w_{p} \\
1 & \text { if } w_{o} Q \geq W+w_{p}\end{cases}
\end{gathered}
$$

Next we formulate the proposed EOQ model as follows

$$
\begin{aligned}
& \max \left\{\mu_{\tilde{O}}(T A C(D, S, Q)) \mu_{\tilde{C}}(S(Q))\right\} \\
& \min \left\{\nu_{\tilde{O}}(T(D, S, Q)), \nu_{\tilde{C}}(S(Q))\right\} \\
& \text { such that } \\
& 0<\mu_{\tilde{O}}(T A C(D, S, Q))+\nu_{\tilde{O}}(T A C(D, S, Q))<1 ; \\
& 0<\mu_{\tilde{C}}(S(Q))+\nu_{\tilde{C}}(S(Q))<1 ; \\
& D, S, Q>0 .
\end{aligned}
$$


By employing the max-additive operator in IF environment, we convert the multi-objective optimization model to a single objective optimization model as follows:

$$
\max V F_{I F A}(D, S, Q)=I_{K}-V F_{I F A 1}(D, S, Q)
$$

such that

$$
D, S>0, Q \in\left[\frac{W+\epsilon_{C}}{w_{0}}, \frac{W+w_{p}}{w_{0}}\right], \operatorname{TAC}(\mathrm{D}, \mathrm{S}, \mathrm{Q}) \in\left[\mathrm{T}_{0}+\epsilon_{\mathrm{O}}, \mathrm{T}_{1}\right],
$$

where $I_{K}=\left(\frac{T_{1}}{T_{1}-T_{0}}+\frac{W+w_{p}}{w_{p}}+\frac{T_{0}+\epsilon_{0}}{T_{1}-T_{0}-\epsilon_{0}}+\frac{W+\epsilon_{c}}{w_{p}-\epsilon_{c}}\right)$ and

$$
V F_{I F A 1}(D, S, Q)=\frac{I_{K 1} S D}{Q}+\frac{I_{K 1} a Q^{2}}{6 D}+I_{K 1} \theta D^{1-x} S^{-1}+I_{K 2} w_{0} Q .
$$

Here

$$
I_{K 1}=\left(\frac{1}{T_{1}-T_{0}}+\frac{1}{T_{1}-T_{0}-\epsilon_{0}}\right) \text { and } I_{K 2}=\left(\frac{1}{w_{p}}+\frac{1}{w_{p}-\epsilon_{c}}\right) .
$$

Now the problem becomes as follows

$$
\min V F_{I F A 1}(D, S, Q)=\frac{I_{K 1} S D}{Q}+\frac{I_{K 1} a Q^{2}}{6 D}+I_{K 1} \theta D^{1-x} S^{-1}+I_{K 2} w_{0} Q
$$

subject to same restriction as in (3)

Next we apply GP to solve model (4). We find that model (4) is an unconstrained PGPP with DD as 0 . The dual problem to model (4) is as follows:

$$
\begin{aligned}
& \max d_{i}(w)=\left(\frac{I_{K 1}}{w_{01}}\right)^{w_{01}}\left(\frac{a I_{K 1}}{6 w_{02}}\right)^{w_{02}}\left(\frac{\theta I_{K 1}}{w_{03}}\right)^{w_{03}}\left(\frac{w_{0} I_{K 2}}{w_{04}}\right)^{w_{04}} \\
& \text { such that } \\
& w_{01}+w_{02}+w_{03}+w_{04}=1 \\
& w_{01}-w_{02}+(1-x) w_{03}=0 \\
& w_{01}-w_{03}=0 \\
& -w_{01}+2 w_{02}+w_{04}=0 \\
& w_{01}, w_{02}, w_{03}, w_{04} \geq 0 .
\end{aligned}
$$

The optimum decision variables are as follows

$$
\begin{aligned}
& D^{*}=\left\{\theta\left(\frac{a}{6(2-x)}\right)^{3}\left(\frac{I_{K 1}(2 x-3)}{I_{K 2} w_{0}}\right)^{5}\right\}^{\frac{1}{x+1}}, \\
& S^{*}=\left\{\theta\left(\frac{I_{K 2} w_{0}}{I_{K 1}(2 x-3)}\right)^{(3 x-2)}\left(\frac{a}{6(2-x)}\right)^{(1-2 x)}\right\}^{\frac{1}{x+1}}, \\
& Q^{*}=\left\{\theta\left(\frac{I_{K 1}(2 x-3)}{I_{K 2} w_{0}}\right)^{(4-x)}\left(\frac{a}{6(2-x)}\right)^{(2-x)}\right\}^{\frac{1}{x+1}}
\end{aligned}
$$

with the optimal TAC being as follows: 


$$
\begin{gathered}
\operatorname{TAC}^{*}\left(D^{*}, S^{*}, Q^{*}\right) \\
=\left[\left\{\theta\left(\frac{a}{6}\right)^{(2-x)}\left(\frac{I K_{1}(2 x-3)}{I k_{2} w_{0}}\right)^{(3-2 x)}\right\}^{\frac{1}{x+1}}\left\{2\left(\frac{1}{2-x}\right)^{\left(\frac{2-x}{x+1}\right)}+\left(\frac{1}{2-x}\right)^{\left(\frac{1-2 x}{x+1}\right)}\right\}\right],
\end{gathered}
$$

provided that $Q^{*} \in\left[\frac{W+\epsilon_{C}}{w_{0}}, \frac{W+w_{p}}{w_{0}}\right], T A C^{*}\left(D^{*}, S^{*}, Q^{*}\right) \in\left[T_{0}+\epsilon_{O}, T_{1}\right]$.

\section{Numerical application}

In this paper, we consider a simple numerical application to solve a proposed model in IF environment as follows.

A manufacturing company produces machines $P B A_{597}$. The inventory carrying cost for the machines is $R s .105$ per unit per year. The production cost of this machine varies inversely with the demand and set-up cost. From the past experiences, we can consider the production cost of the machine $P B A_{597}$ at about $120 D^{-0.75} S^{-1}$, where $D$ is the demand rate and $S$ is the set-up cost. The company has storage space area per unit time $\left(w_{0}\right)$ and total storage space area $(W)$ as 100 sq. ft. and 2000 sq. ft., respectively. The task is to determine the optimal demand rate $(D)$, set-up cost $(S)$, production quantity $(Q)$ and, hence, optimal TAC of the production system.

Hence, the mathematical model is of the form

$$
\begin{aligned}
& \text { Min } T A C(D, S, Q)=\frac{S D}{Q}+\frac{105 Q^{2}}{6 D}+120 D^{-0.75} S^{-1} \\
& \text { subject to } \\
& S(Q) \equiv 100 Q \leq 2000 \\
& D, S, Q>0
\end{aligned}
$$

The goal and goal plus tolerance values for TAC and space constraint are presented in Table 2.

\begin{tabular}{|c|c|c|c|c|}
\hline & Demand $(D)$ & Set-up Cost $(S)$ & $\begin{array}{c}\text { Production } \\
\text { quantity }(Q)\end{array}$ & $\begin{array}{c}\text { Total average cost } \\
(\mathrm{TAC}(D, S, Q))\end{array}$ \\
\hline Goal & 4047.477 & 0.034 & 20.000 & 15.565 \\
\hline $\begin{array}{l}\text { Goal plus } \\
\text { tolerance }\end{array}$ & 5521.645 & 0.028 & 23.000 & 15.089 \\
\hline
\end{tabular}

Table 2: Goal and goal plus tolerance values

Based on these values, we construct the following linear membership and non-membership functions of TAC and space constraint:

$$
\mu_{\tilde{O}}(T A C(D, S, Q))= \begin{cases}1 & \text { if } T A C(D, S, Q) \leq 15.089 \\ \frac{15.565-T A C(D, S, Q)}{0.476} & \text { if } 15.089 \leq T A C(D, S, Q) \leq 15.565 \\ 0 & \text { otherwise. }\end{cases}
$$




$$
\begin{aligned}
& \mu_{\tilde{C}}(S(Q))= \begin{cases}1 & \text { if } 100 Q \leq 2000 \\
\frac{2300-100 Q}{300} & \text { if } 2000 \leq 100 Q \leq 2300 \\
0 & \text { otherwise. }\end{cases} \\
& \nu_{\tilde{O}}(T A C(D, S, Q))= \begin{cases}0 & \text { if } T A C(D, S, Q) \leq 15.189 \\
\frac{T A C(D, S, Q)-15.189}{0.376} & \text { if } 15.189 \leq T A C(D, S, Q) \leq 15.565 \\
1 & \text { otherwise. }\end{cases} \\
& \nu_{\tilde{C}}(S(Q))= \begin{cases}0 & \text { if } 100 Q \leq 2050 \\
\frac{100 Q-2050}{250} & \text { if } 2050 \leq 100 Q \leq 2300 \\
1 & \text { if } 100 Q \geq 2300\end{cases}
\end{aligned}
$$

Therefore, we obtain the single objective EOQ model with space constraint as follows

$$
\begin{aligned}
& \text { Min } T A C(D, S, Q)=\frac{4.76 S D}{Q}+\frac{83.3 Q^{2}}{D}+571.2 D^{-0.75} S^{-1}+73.3 Q \\
& \text { such that } \\
& D, S>0, Q \in[20.5,23], \operatorname{TAC}(D, S, Q)=[15.089,15.565]
\end{aligned}
$$

We solve the model (6) by the GP technique and we obtain the optimal solution as follows

$$
\begin{gathered}
w_{01}^{*}=0.364, w_{02}^{*}=0.091, w_{03}^{*}=0.364, w_{04}^{*}=0.182 . \\
D^{*}=4998.630, S^{*}=0.030, Q^{*}=21.993, T A C^{*}\left(D^{*}, S^{*}, Q^{*}\right)=15.240 .
\end{gathered}
$$

Here we note that the optimal TAC is 15.240 units with demand as 4998.630 units, set-up cost as 0.030 units and production quantity as 21.993 units. Next we compare the relative performance of proposed model by comparing its result with that obtained by employing crisp GP and fuzzy GP and present it in Table 3. We find that the optimal TAC is more preferable in IF environment than that of the crisp and fuzzy environment. Further, the IF environment yields higher demand for the machine $P B A_{597}$ with lower set-up cost. Furthermore, the production quantity increases in IF environment.

\begin{tabular}{|c|c|c|c|c|}
\hline Geometric programming & Demand $(D)$ & Set-up Cost $(S)$ & $\begin{array}{c}\text { Production } \\
\text { quantity }(Q)\end{array}$ & $\begin{array}{c}\text { Total average cost } \\
\text { (TAC }(D, S, Q))\end{array}$ \\
\hline Crisp environment & 4047.477 & 0.034 & 20.000 & 15.565 \\
\hline Fuzzy environment & 4742.869 & 0.031 & 21.479 & 15.320 \\
\hline Intuitionistic Fuzzy environment & 4998.630 & 0.030 & 21.993 & 15.240 \\
\hline
\end{tabular}

Table 3: Comparison of optimal solutions 


\subsection{Sensitivity analysis}

In this paper, we investigate the optimal policy of DM for a proposed model in real life based IF environment. Here we perform a sensitivity analysis of following key parameters:

(i) storage space per machine $\left(w_{0}\right)$ (Table 4$)$;

(ii) shape parameter $(x)$ (Table 5);

(iii) variational parameter $(a)$ (Table 6);

(iv) shape parameter $(\theta)$ (Table 7)

and present corresponding optimal solutions in IF environment.

We present the phenomenon of change of storage space per machine $\left(w_{0}\right)$ in Table 4 . We observe that the optimal TAC is most preferable to DM in IF environment among said environments, which is well explained in Fig. 6. Also we find that each reduction in storage space per machine reduces TAC not only in IF environment but also in other environments. Hence, the management should trim down the packet size of finished goods so as to cut TAC.

\begin{tabular}{|c|c|c|c|c|c|}
\hline TAC & 80 & 90 & 100 & 110 & 120 \\
\hline Crisp environment & 14.812 & 15.205 & 15.565 & 15.898 & 16.209 \\
\hline Fuzzy environment & 14.718 & 15.032 & 15.320 & 15.595 & 15.840 \\
\hline Intuitionistic fuzzy environment & 14.513 & 14.826 & 15.240 & 15.379 & 15.623 \\
\hline
\end{tabular}

Table 4: Sensitivity analysis of storage space per machine $\left(w_{0}\right)$

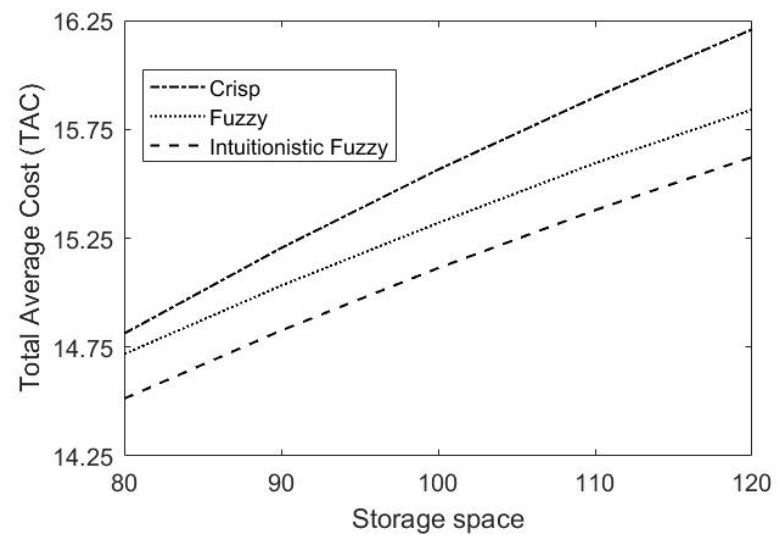

Figure 6: Effect on TAC due to alteration of storage space per machine $\left(w_{0}\right)$

Next we consider the change of shape parameter $(x)$ in Table 5. Here we find that optimal TAC rapidly decreases for every increment in value of shape parameter and Hence, for every rise in demand in each of the said environments. It is consistent with common knowledge. Also in nearly all cases, we get most preferable optimal TAC in IF environment, which can be observed in Fig. 7.

Again we perform sensitivity analysis of variational parameter $(a)$ and present it in Table 6 . Here for all time, we obtain best possible TAC in the IF environment among said environments, which can be visualized in Fig. 8. Also in all of these environments, the optimal TAC reduces as holding cost decreases. 


\begin{tabular}{|c|c|c|c|c|c|}
\hline TAC & 1.6 & 1.7 & 1.75 & 1.8 & 1.9 \\
\hline Crisp environment & 25.799 & 18.612 & 15.565 & 12.860 & 8.385 \\
\hline Fuzzy environment & 26.582 & 18.554 & 15.320 & 12.585 & 8.408 \\
\hline IF environment & 26.429 & 18.349 & 15.240 & 12.385 & 8.198 \\
\hline
\end{tabular}

Table 5: Sensitivity analysis of shape parameter $(x)$

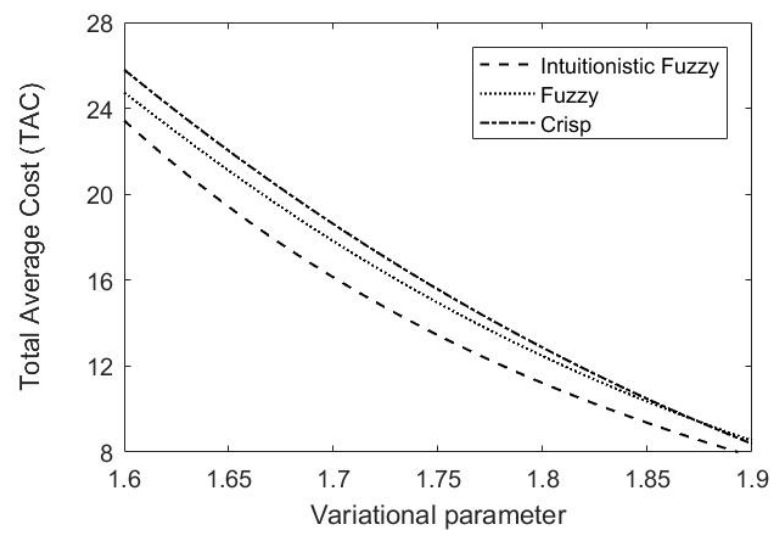

Figure 7: Effect on TAC due to alteration of shape parameter $(x)$

\begin{tabular}{|c|c|c|c|c|c|}
\hline TAC & 95 & 100 & 105 & 110 & 115 \\
\hline Crisp environment & 15.393 & 15.481 & 15.565 & 15.646 & 15.723 \\
\hline Fuzzy environment & 15.182 & 15.253 & 15.320 & 15.387 & 15.448 \\
\hline IF environment & 15.103 & 15.174 & 15.240 & 15.307 & 15.368 \\
\hline
\end{tabular}

Table 6: Sensitivity analysis of variational parameter $(a)$

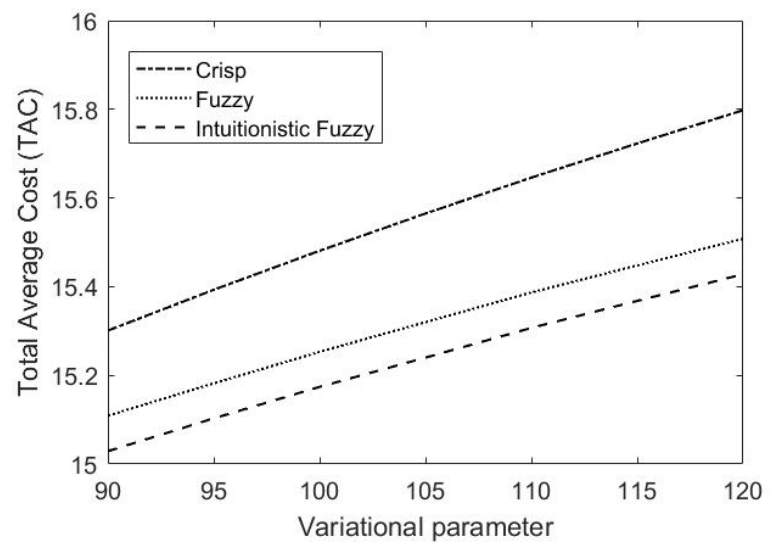

Figure 8: Effect on TAC due to alteration of variational parameter $(a)$

Next we consider the change of the shape parameter $(\theta)$ and present the result in Table 7 . As before, we find that the optimal TAC is favorable to DM in the IF environment among said environments. Fig. 9 brings clarity to this phenomenon. Additionally, we observe that the optimal $\mathrm{TAC}$ can be further reduced by decreasing the value of the shape parameter. 


\begin{tabular}{|c|c|c|c|c|c|}
\hline TAC & 100 & 110 & 120 & 130 & 140 \\
\hline Crisp environment & 14.354 & 14.975 & 15.565 & 16.129 & 16.669 \\
\hline Fuzzy environment & 14.338 & 14.843 & 15.320 & 15.773 & 16.037 \\
\hline IF environment & 14.146 & 14.643 & 15.240 & 15.560 & 15.985 \\
\hline
\end{tabular}

Table 7: Sensitivity analysis of shape parameter $(\theta)$

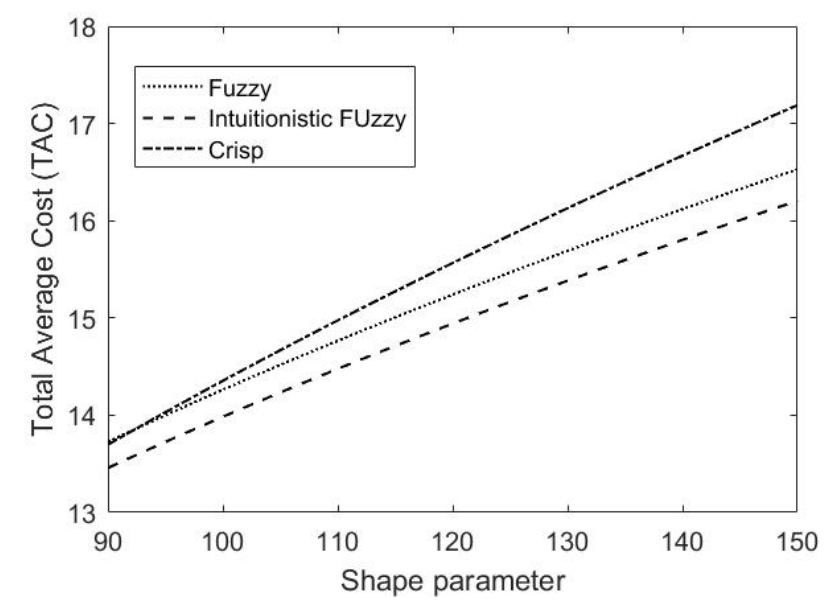

Figure 9: Effect on TAC due to alteration of shape parameter $(\theta)$

\section{Conclusions}

In this paper, we consider a deterministic single objective EOQ model with space constraint and solve it in IF environment. It is well-known that fuzzy sets can better represent real life cases than crisp sets. In recent years, several generalizations of fuzzy set theory have appeared. One of them is IF set, where one considers both level of membership and level of non-membership of the elements of universal set.

On the other hand, the advantage of GP is manifold. As per Cao, GP provides us with a systematic approach for solving a class of nonlinear optimization problems by determining optimal values of decision variables and objective functions. Whereas existing literature survey finds that GP is used in fuzzy environment, we find very few papers, where EOQ models with space constraint were solved by GP in IF environment. In this paper, we formally introduce GP in IF environment. Here we employ max-additive operator to convert EOQ model with space constraint to single objective PGPP and thereby solve it in proposed IF GP. In a numerical application, we find that the optimal solution, obtained in IF GP is more preferable to the decision maker than that obtained in the crisp or fuzzy GP. In addition, we have performed sensitivity analysis of key parameters of the proposed model. And we have listed several key managerial insights, which were further explained graphically.

\section{Future scopes of research}

We locate lots of scopes for further improvement of proposed model. We list some of them as follows: 
(i) We can consider multiple products. In that case, we can employ modified GP in IF environment.

(ii) The shape parameters can be IF in nature.

(iii) We can allow shortage or partial backlogging of items in inventory and update the model accordingly.

(iv) We can use other optimization methods to solve non-linear models in IF environment.

(v) And last but not the least, we can discuss the present model in other imprecise environments.

\section{References}

[1] Angelov, P. P. (1997). Optimization in intuitionistic fuzzy environment, Fuzzy Sets and Systems, 86(3), 299-306.

[2] Atanassov, K. (1986). Intuitionistic fuzzy sets, Fuzzy Sets ans Systems, 20(1), 87-96.

[3] Beightler, C. S., \& Phillips, D. T. (1976). Applied geometric programming, John Wiley \& Sons, New York.

[4] Bellman, R. E., \& Zadeh, L. A. (1970). Decision-making in a fuzzy environment, Management Sciences, 17(4), 141-164.

[5] Chakrabortty, S., Pal, M., \& Nayak, P. K. (2013). Intuitionistic fuzzy optimization technique for Pareto optimal solution of manufacturing inventory models with shortages, European Journal of Operational Research, 228(2), 381-387.

[6] Cheng, T. C. E. (1989). An economic order quantity model with demand-dependent unit cost, European Journal of Operational Research, 40(2), 252-256.

[7] Cheng, T. C. E. (1991). An economic order quantity model with demand-dependent unit production cost and imperfect production process, IIE Transactions, 23(1), 23-28.

[8] Dey, S., \& Roy, T. K. (2014). Optimized solution of two bar truss design using intuitionistic fuzzy optimization technique, International Journal of Information Engineering and Electronic Business, 4, 45-51.

[9] Duffin, R. J. (1962). Cost minimization problems treated by geometric means, Operations Research, 10(5), 668-675.

[10] Duffin, R. J., Peterson, E. L., \& Zener, C. M. (1967) Geometric programming, John Wiley, New York.

[11] Garai, A., Mandal, P., \& Roy, T. K. (2016). Interactive intuitionistic fuzzy technique in multi-objective optimization, International Journal of Fuzzy Computation and Modelling, 2(1), 1-14. 
[12] Harris, F. M. (1913). How many parts to make at once, Factory, The Magazine of Management, 10(2), 135-136.

[13] Islam, S. (2008). Multi-objective marketing planning inventory models: A geometric programming approach, Applied Mathematics and Computation, 205(1), 238-246.

[14] Islam, S., \& Mandal, W. A. (2017). A fuzzy inventory model (EOQ Model) with unit production cost, time depended holding cost, without shortages under a space constraint: A fuzzy parametric geometric programming (FPGP) approach, Independent Journal of Management \& Production (IJM\&P), 8(2), 299-318.

[15] Jana, B., \& Roy, T. K. (2007). Multi-objective intuitionistic fuzzy linear programming and its application in transportation model, Notes on Intuitionistic Fuzzy Sets, 13(1), 34-51.

[16] Janseen, L., Claus, T., \& Sauer, J. (2016). Literature review of deteriorating inventory models by key topics from 2012 to 2015, International Journal of Production Economics, $182,86-112$.

[17] Jung, H., \& Klein, C. M. (2005). Optimal inventory policies for an economic order quantity model with decreasing cost functions, European Journal of Operational Research, 165(1), $108-126$.

[18] Jung, H., \& Klein, C. M. (2006). Optimal inventory policies for profit maximizing EOQ models under various cost functions, European Journal of Operational research, 174(2), 689-705.

[19] Kaur, P., \& Rachana, K. N. L. (2016) An intuitionistic fuzzy optimization approach to vendor selection problem, Perspective in Science, 8, 348-350.

[20] Kochenberger, G. A. (1971). Inventory models: optimization by geometric programming, Decision Sciences, 2(2), 193-205.

[21] Lee, W. J. (1994). Optimal order quantities and prices with storage space and inventory investment limitations, Computers \& Industrial Engineering, 26(3), 481-488.

[22] Mahapatra, G. S., Mandal, T. K., \& Samanta, G. P. (2013) EPQ model with fuzzy coefficient of objective and constraint via parametric geometric programming, International Journal of Operational Research, 17(4), 436-448.

[23] Mandal, N. K., Roy, T. K., \& Maiti, M. (2006). Inventory model of deteriorated items with a constraint: A geometric programming approach, European Journal of Operational Research, 173(1), 199-210.

[24] Nezami, F. G., Aryanezhad, M. B., \& Sadjadi, S. J. (2009). Determining optimal demand rate and production decisions: A geometric programming approach , World Academy of Science, Engineering and Technology International Journal of Mechanical, Aerospace, Industrial, Mechatronic and Manufacturing Engineering, 3(1), 55-60. 
[25] Park, K. S. (1987). Fuzzy-set theoretic interpretation of economic order quantity, IEEE Transactions on Systems, Man, and Cybernetics, 17(6), 1082-1084.

[26] Pramanik, S., \& Roy, T. K. (2005). An intuitionistic fuzzy goal programming approach to vector optimization problem, Notes on Intuitionistic Fuzzy Sets, 11(5), 1-14.

[27] Roy, T. K., \& Maiti, M. (1997). A fuzzy EOQ model with demand-dependent unit cost under limited storage capacity, European Journal of Operational Research, 99(2), 425-432.

[28] Sadjadi, S. J., Aryanezhad, M. B., \& Jabbarzadeh, A. (2010). Optimal marketing and production planning with reliability consideration, African Journal of Business Management, 4(17), 3632-3640.

[29] Sadjadi, S. J., Hesarsorkh, A. H., Mohammadi, M., \& Naeini, A. N. (2015). Joint pricing and production management:a geometric programming approach with consideration of cubic production cost function, Journal of Industrial Engineering International, 11(2), 209-223.

[30] Sommer, G. (1981). Fuzzy inventory scheduling, in applied systems, Applied Systems and Cybernetics, G. Lasker Edition, Academic Press, New York, 16(6), 3052-3062.

[31] Tabatabaei, S. R. M., Sadjadi, S. J., \& Makui, A. (2017). Optimal pricing and marketing planning for deteriorating items, PLOS ONE, 12(3), 1-21.

[32] Tanaka, H., Okuda, T., \& Asai, K. (1974). On fuzzy-mathematical programming, Journal of Cybernetics, 3(4), 37-46.

[33] Zadeh, L. A. (1965). Fuzzy sets, Information and Control, 8(3), 338-353.

[34] Zener, C. M. (1961). A mathematical aid in optimizing engineering design, Proceedings of the National Academy of Sciences of the United States of America, 47(4), 537-539.

[35] Zener, C. M. (1962). A further mathematical aid in optimizing engineering design, Proceedings of the National Academy of Sciences of the United States of America, 48(4), 518-522.

[36] Zimmerman, H. J. (1976). Description and optimization of fuzzy systems, International Journal of General Systems, 2(1), 209-215. 